\title{
La disponibilidad de alimentos en México: un análisis de la producción agrícola de 35 años y su proyección para 2050
}

\author{
Anacleto Sosa Baldivia y Guadalupe Ruíz Ibarra \\ Nutrilite-Amway, México e Instituto Tecnológico Superior de Tamazula \\ de Gordiano, México
}

Resumen

En los próximos 35 años el mayor reto que enfrentará la agricultura mexicana es asegurar el abasto de alimentos para la población de nuestro país. El objetivo de este trabajo fue analizar la producción agrícola en México en los últimos 35 años y su proyección para 2050. De 1980 a 2015, la población mexicana creció 67 por ciento, para cubrir sus requerimientos alimenticios, la producción agrícola incrementó 117 por ciento a través de aumentar 67 por ciento el rendimiento de los cultivos y 23 por ciento el área cultivada. Entre 1980 y 2015 la tierra disponible per cápita disminuyo 31 por ciento, esto significa que en el futuro para aumentar la producción los agricultores deberán enfocarse a mejorar el rendimiento de los cultivos. La ganancia en rendimiento lograda en cereales, hortalizas, frutales, forrajes y cultivos industriales en el periodo analizado indica que es posible asegurar su abasto por esta vía. No obstante, debido a que en especies leguminosas y oleaginosas, la mejora del rendimiento ha sido escasa, en el corto plazo se necesitará invertir en investigación e infraestructura agrícola que ayude a mejorar su productividad. Los resultados obtenidos sugieren que la agricultura de México será capaz de producir alimentos que a futuro demande la población, sin embargo para asegurar su abasto, también será necesario mejorar su acceso y lo más importante distribuirlos con equidad.

Palabras clave: Población, alimentación, agricultura, cereales, leguminosas.

\section{Abstract}

The availability of food in Mexico: an analysis of 35 years agricultural production and its projection for 2050

Over the next 35 years, the greatest challenge which will face Mexican agriculture is to ensure the food supply for the population of México. The objective of this work was to analyze the agricultural production in Mexico in the last 35 years and its projection for 2050. From 1980 to 2015, the Mexican population grew by 67 por ciento, to meet their food demand, the agricultural production increased 117 por ciento through to increase 67 por ciento the crop yield and 23 por ciento the cultivated area. Between 1980 and 2015 the land available per capita decreased 31 por ciento, this means that in the future to increase the agricultural production, the farmers must focus in improving the crop yield. The gain yield achieved in cereals, vegetables, fruit, forages and industrial crops during the analyzed period indicates that in a future it is possible to ensure its supply for this way. However, since on legumes and oilseeds species the gain yields on the past has been scarce, on the short term, there will be needed to invest in research and agricultural infrastructure that will help to improve your productivity. These results suggest that farmers in Mexico will be able to produce the food that future demands the population, however to ensure its supply, there will also be necessary to improve their access and most importantly distribute them fairly.

Key words: population, food, agriculture, cereals, legumes. 


\section{INTRODUCCIÓN}

nivel mundial, el mayor reto que la agricultura enfrentara en
los próximos 35 años es asegurar el abasto de alimentos para la
población, sin embargo su magnitud estará en función de la tasa naturales de cada país. De acuerdo con la FAO (2009), para alimentar una población de 9,100 millones de habitantes, entre 2007 y 2050, el volumen de la producción agrícola mundial debe aumentar 70 por ciento; esto significa que la superficie cultivada debe incrementar 70 millones ha más de las actuales ( 1500 millones ha) y el rendimiento de los cultivos tomando como referencia los cereales debe incrementar de 1.2 a 4.3 ton/ha. En el ámbito nacional, la CONAPO (2006) estima que para el año 2050, la población incrementara 17 por ciento con respecto a la actual (119.5 millones de habitantes) (INEGI, 2015a); este aumento demandará servicios de vivienda, salud, energía eléctrica, agua potable y desde luego un suministro continuo de alimentos (Azuz-Adeath y Rivera-Arriaga, 2007; FAO, 2013). El asegurar el abasto de alimentos para los próximos años en México será un gran reto ya que aun cuando el área cultivable actual es 27.8 millones ha (INEGI-SAGARPA, 2015) se estima que esta superficie podría disminuir por el cambio de uso de suelo para construir viviendas. Considerando esta situación, para aumentar la producción agrícola, más que abrir nuevas tierras al cultivo, los productores se deben enfocar a mejorar el rendimiento por unidad de superficie. De acuerdo con Agrios (1998), para lograr esto, se necesita incorporar a la agricultura todas las prácticas agronómicas que ayudan a incrementar el rendimiento dentro de las que se incluye el uso de: (1) cultivares mejorados; (2) fertilizantes; (3) irrigación, (4) pesticidas para controlar malezas, plagas y enfermedades; (5) rotación de cultivos; y (6) sistemas de labranza. Además debe emplearse la hidroponía (Resh, 1992) y la agricultura protegida (Castellanos-Ramos y Borbón-Morales, 2009) como técnicas que permitan intensificar el uso de la tierra cultivable (Turrent-Fernández y Moreno-Dahme, 1998). Al respecto la pregunta que surge es: ¿Cuál es la situación actual de la agricultura nacional? una frase famosa menciona que para entender el presente y predecir el futuro es necesario conocer el pasado. Con base en lo anterior, el objetivo de este trabajo fue determinar que ha ocurrido con la agricultura de México en los últimos 35 años, su estado actual y cuál es su perspectiva para el futuro con relación a la producción de alimentos. 


\section{Materiales Y MÉtodos}

Para realizar esta investigación, se utilizó las bases de datos oficiales del SIAP (2016) y los informes del censo de población y vivienda (INEGI 2015a); el censo agrícola y ganadero (INEGI-SAGARPA, 2015); y la balanza comercial de productos agropecuarios para el 2015 en México (INEGI, 2015b). El primer indicador estimado fue la disponibilidad de tierra per cápita, para esto el área sembrada se dividió entre el número de habitantes. De 1980 a 2015, los cálculos se hicieron por lustro; pero después de esta fecha y hasta 2050 se hicieron cada diez años. Debido a que en México se producen alrededor de 264 especies vegetales (SIAP, 2016), previo al análisis todos estos cultivos se clasificaron dentro de ocho grupos que incluyó cereales (CER), leguminosas (LEG), oleaginosas (OLE), hortalizas (HOR), frutales (FRU), forrajes (FOR) y aromáticas y medicinales (AROyMED). Las especies ornamentales por carecer de importancia alimenticia aunque también se agruparon no se incluyeron en el análisis. A los ocho grupos de cultivos estudiados se les calculó su volumen de producción, área sembrada y rendimiento por unidad de superficie por lustro. Adicionalmente, se realizó un análisis de correlación entre los tres indicadores de la producción antes mencionados, y el crecimiento poblacional ocurrido a través del tiempo. La disponibilidad teórica de alimentos se estimó dividiendo el volumen de la producción de cada grupo entre el número de habitantes para el año 2010. Posteriormente, este parámetro se utilizó para estimar la demanda de alimentos proyectada la cual se obtuvo multiplicando la disponibilidad teórica de alimentos de 2010 por el número de habitantes que la CONAPO (2006) estimó para 2015-2050 para el escenario de crecimiento máximo. Para el cálculo del incremento del rendimiento requerido para asegurar a futuro el abasto de los grupos de cultivos analizados, se consideró que la producción es función exclusiva del rendimiento; bajo este supuesto, este parámetro se calculó dividiendo la demanda de alimentos proyectada para cada grupo entre su superficie sembrada en 2010 (SIAP, 2016). La ganancia de rendimiento exhibida en los ocho grupos de cultivos analizados en los últimos 35 años se obtuvo mediante un análisis de regresión lineal en donde la variable dependiente (y) fue el rendimiento expresado $\mathrm{kg} \mathrm{ha}^{-1} \mathrm{y}$ la variable independiente (x) fue el tiempo definido en años. Una vez generada la ecuación, se tomó como ganancia de rendimiento anual para cada grupo de cultivos estudiado al valor de la pendiente estimada (expresada en $\mathrm{kg} / \mathrm{ha} / \mathrm{año}$ ). Cabe señalar que en los cálculos realizados se consideró que toda la producción se destina a 
satisfacer la demanda nacional, en la realidad esto no ocurre ya que México como el resto del mundo exporta e importa de alimentos.

\section{RESUltAdOS Y DISCUSIÓN}

\section{Incremento poblacional y disponibilidad de tierra}

En los últimos 35 años la población mexicana incrementó 79 por ciento pasando de 66.8 millones en 1980 a 119.5 millones en 2015 (Tabla 1). En este mismo periodo, la superficie agrícola creció 22 por ciento y las 17.99 millones ha sembradas en 1980 aumentaron hasta ser 22.2 millones ha en 2015. Aun cuando la superficie cultivada incrementó, la tierra disponible per cápita estimada para 1980 ( 0.27 ha/habitante) sólo se mantuvo sin cambio hasta 1985, y posteriormente decreció hasta ser 0.18 ha/habitante en 2015; esto significa que en un lapso de 35 años, la tierra agrícola disponible per cápita disminuyo en 31 por ciento. El escenario que se proyecta para el futuro es más alarmante ya que considerando que para el 2050 seremos 140 millones de habitantes (CONAPO, 2006), la tierra agrícola disponible podría disminuir a 0.16 ha/habitante/año. Considerando esta proyección la pregunta que surge es: ¿estamos preparados para enfrentar este reto?

Tabla 1: Incremento de la población, superficie sembrada y disponibilidad per cápita de tierra cultivable en México

\begin{tabular}{lrrr}
\hline Año & $\begin{array}{r}\text { Población } \\
\text { Millones habitantes }\end{array}$ & $\begin{array}{r}\text { Superficie cultivable } \\
\text { Millones ha }\end{array}$ & $\begin{array}{r}\text { Disponibilidad tierra } \\
\text { Ha/habitante }\end{array}$ \\
\hline 1980 & 66.8 & 17.99 & 0.27 \\
1985 & 74.0 & 20.16 & 0.27 \\
1990 & 81.2 & 19.73 & 0.24 \\
1995 & 91.2 & 20.94 & 0.23 \\
2000 & 97.5 & 21.78 & 0.22 \\
2005 & 103.2 & 21.64 & 0.21 \\
2010 & 112.3 & 21.95 & 0.20 \\
2015 & 119.5 & 22.2 & 0.18 \\
Incremento & 52.7 & 3.96 & -0.08 \\
$\%$ & 78.9 & 22.2 & -31.0 \\
Crecimiento poblacional proyectado & & \\
2020 & 118.2 & 22.2 & 0.18 \\
2030 & 127.5 & 22.2 & 0.17 \\
2040 & 135.0 & 22.2 & 0.16 \\
2050 & 140.0 & 21.95 & 0.16 \\
\hline
\end{tabular}

Nota: los valores negativos significan que hubo disminución del parámetro.

Fuente: los cálculos se realizaron con base en la información del INEGI (2015b), SIAP (2017) y la CONAPO (2006). 


\section{Estado actual de la agricultura}

México a diferencia de otros países cuenta con una gran diversidad de condiciones edafoclimáticas que le permiten cultivar 264 especies vegetales (SIAP, 2016); como resultado de esto, la alimentación del pueblo mexicano se basa en productos de origen vegetal en donde el maíz, frijol y chile conforman la base de la dieta (Román et al., 2013). Además, hoy con 5.8 millones de toneladas de productos agrícolas exportadas a otros países, México es el cuarto exportador mundial de frutas y hortalizas tan solo superado por Holanda, España y China (Financiera Rural, 2008; Macías, 2010). De acuerdo con la información presentada en la Tabla 2, de los ocho grupos vegetales cultivados en México, CER, FOR y LEG cubren 65 por ciento del área agrícola; esto no sorprende ya que desde tiempos ancestrales, CER y LEG son la base de la alimentación en México (MuñozVelázquez et al., 2009), y actualmente, aun cuando 202 especies se explotan con fines alimentarios, son maíz y frijol los que cubren 44 por ciento del área cultivada (8.015 y 1.774 millones de hectáreas, respectivamente). Los FOR representan 21 por ciento del área de cultivo y estos aunque no se consumen directamente, su alta demanda se asocia a que se usan como alimento en la producción de leche, carne y huevos. Desde 1980 la demanda de alimentos de origen animal ha incrementado (Martínez-Jasso y Villezca-Becerra, 2005); por ello, no sorprende que los FOR cubran una área mayor a la que se destina para la producción de especies LEG y OLE. El área cultivada de LEG desde 1980 a la fecha ha permanecido sin cambios, e incluso la de OLE ha disminuido. En contraparte, las HOR a pesar de representar una superficie relativamente pequeña ( 0.67 millones de hectáreas) y que es muy similar a la que se destina a la producción de trigo, son clave en la economía ya que la exportación de varias de las especies de este grupo es parte de una estrategia económica para generar divisas y empleos. Esta estrategia se implementó en la década de los setentas cuando México todavía era autosuficiente en la producción de CER; esto resulto en que hoy exportemos HOR y FRU; pero también importemos CER y OLE (Borlaug et al., 1982). La importación de CER y OLE que realiza México, a muchos hace pensar que tiene un déficit de alimentos; pero ¿qué tan cierto es esto? La respuesta a esta pregunta sin especular se puede obtener estudiando cómo ha crecido la población Mexicana y cuál es su relación con la producción de alimentos de origen vegetal. 


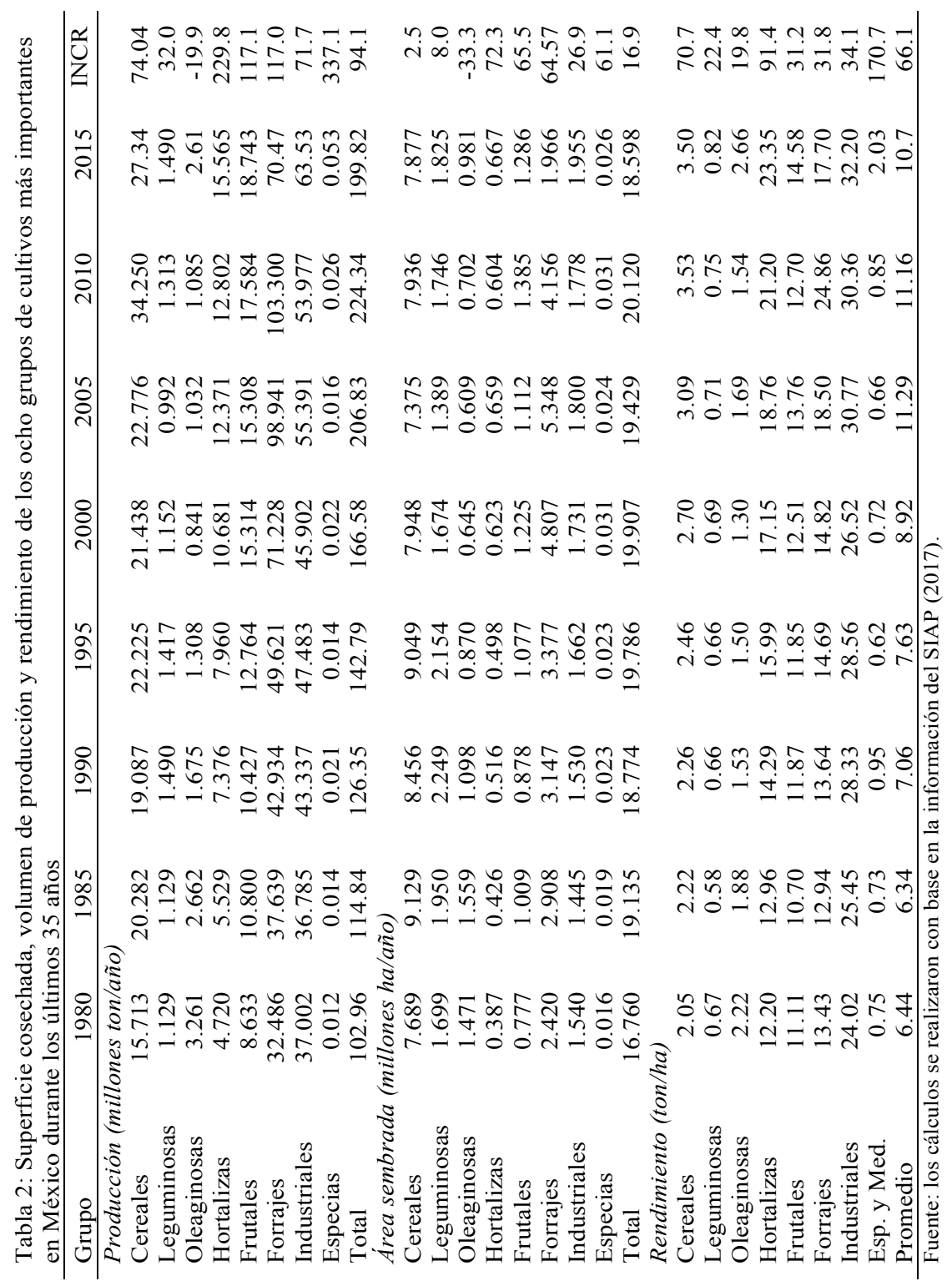




\section{Producción agrícola y crecimiento poblacional}

Como se puede apreciar en la Tabla 2, de 1980 a 2015 la producción agrícola en México incrementó 94 por ciento pasando de 102.96 a 199.94 millones de toneladas de alimentos. Debido a que en este periodo, la producción de alimentos vegetales creció a una tasa mayor que la población, la disponibilidad de alimentos vegetales per cápita incrementó en 12 por ciento (Tabla 3). Como resultado de esto, la disponibilidad de alimentos por persona en 2015 fue 177 kilos mayor que la de 1980, siendo en los CER, HOR, FRU y FOR los que registraron mayor contribución.

Tabla 3: Disponibilidad de alimentos per cápita en México (1980-2015)

\begin{tabular}{lrrrrrrrr}
\hline Grupo & 1980 & 1985 & 1990 & 1995 & 2000 & 2005 & 2010 & 2015 \\
\hline kg/habitante año & & & & & & & \\
Cereales & 228.4 & 274.1 & 235.1 & 243.7 & 219.9 & 220.7 & 316.0 & 228.8 \\
Leguminosas & 16.4 & 15.3 & 18.3 & 15.5 & 11.8 & 9.6 & 12.1 & 12.5 \\
Oleaginosas & 47.4 & 36.0 & 20.0 & 14.3 & 8.6 & 10.0 & 10.0 & 21.9 \\
Hortalizas & 68.6 & 74.7 & 90.8 & 87.3 & 109.5 & 119.9 & 118.1 & 130.3 \\
Frutales & 125.5 & 145.9 & 128.4 & 140.0 & 157.1 & 148.3 & 162.2 & 156.8 \\
Forrajes & 472.2 & 508.6 & 528.7 & 544.1 & 730.5 & 958.7 & 953.0 & 589.8 \\
Industriales & 537.8 & 497.1 & 533.7 & 520.6 & 470.8 & 536.7 & 497.9 & 531.7 \\
Especias & 0.2 & 0.2 & 0.3 & 0.2 & 0.2 & 0.2 & 0.2 & 0.4 \\
Total & 1496 & 1552 & 1556 & 1566 & 1079 & 2004 & 2178 & 1672 \\
\hline
\end{tabular}

Fuente: los cálculos se realizaron con base en los volúmenes de producción reportados por el SIAP (2017) y el crecimiento poblacional que reporta el INEGI (2015a).

El aumento de disponibilidad de alimentos en el periodo analizado se reflejó en un incremento de la energía per cápita disponible, la cual pasó de $2750 \mathrm{Kcal} /$ persona/día en 1980 a $3146 \mathrm{Kcal} /$ persona/día en 2009 (Moreno-Altamirano et al., 2014; Urquiza-Fernández, 2014). De forma general, la mejora en la disponibilidad de alimentos vegetales resulto de un incremento el rendimiento por unidad de superficie de 66 por ciento y de incrementar su área de producción en 17 por ciento; por ello, de 1980 a 2015 el área cultivada aumento en 1.84 millones hectáreas y el rendimiento medio de todos los cultivos en 4.3 ton/ha. Esta tendencia es congruente con lo que ha ocurrido en varios países de Latinoamérica en donde el aumento de la producción agrícola es atribuido a la gran mejora realizada en la productividad de los cultivos (FAO, 2014). La alta correlación estimada entre la producción total de alimentos vegetales y el crecimiento poblacional $(\mathrm{r}=0.93)$ sugiere que el aumento de la producción agrícola entre 19802015 fue realizado principalmente para satisfacer la demanda de alimentos 
de la población Mexicana (Tabla 4). Aun cuando en volumen de la producción de alimentos vegetales total se incrementó ampliamente durante el periodo estudiado, este un fue proporcional en los ocho grupos de cultivos analizados y fue en CER, HOR, FRU, FOR, IND y AROyMED en donde el volumen producido aumento significativamente $(74,230,117,72,117 \mathrm{y}$ 337 por ciento, respectivamente).

Tabla 4: Asociación del volumen de producción (r) con el aumento poblacional, área cosechada y el rendimiento de ocho grupos de cultivos producidos en México entre 1980-2015

\begin{tabular}{lrrr}
\multicolumn{1}{c}{ Grupo } & \multicolumn{1}{c}{ Población } & \multicolumn{1}{c}{ Área cosechada } & \multicolumn{1}{c}{ Rendimiento } \\
\hline Coeficiente de correlación (r) & & \\
Cereales & $0.93^{*}$ & $-0.14 \mathrm{~ns}$ & $0.94^{*}$ \\
Leguminosas & $0.28 \mathrm{~ns}$ & $0.76^{*}$ & $0.34 \mathrm{~ns}$ \\
Oleaginosas & $-0.47 \mathrm{~ns}$ & $0.91^{* *}$ & $0.80^{* *}$ \\
Hortalizas & $0.99^{* *}$ & $0.95^{* *}$ & $0.98^{* *}$ \\
Frutales & $0.98^{* *}$ & $0.95^{* *}$ & $0.86^{*}$ \\
Forrajes & $0.80^{*}$ & $0.98^{*}$ & $0.93^{* *}$ \\
Industriales & $0.95^{* *}$ & $0.92^{* *}$ & $0.94^{* *}$ \\
AROyMED & $0.48 \mathrm{~ns}$ & $0.77^{*}$ & $0.99^{*}$ \\
Todos los grupos & $0.93^{* *}$ & $0.74^{*}$ & $0.88^{* *}$ \\
\hline *y** Significativo al 0.05 y 0.01 probabilidad, respectivamente, ns = no significativo. \\
\multicolumn{4}{l}{ Fuente: Los valores se estimaron con base en la información de las Tablas 2 y 3. }
\end{tabular}

La correlación estimada entre el volumen de producción de CER, HOR, FRU, FOR e IND y el aumento poblacional ( $\mathrm{r}=0.93,0.99,0.98$, 0.80 y 0.97 , respectivamente) indica que el aumento de su producción fue la respuesta del sector agrícola para cubrir la demanda de alimentos. En contraparte, la producción de LEG incrementó a una tasa menor que el crecimiento poblacional ( 32 por ciento) y en OLE, incluso disminuyo en 20 por ciento, como resultado de esto, ambos grupos no presentaron asociación con el aumento demográfico $(\mathrm{r}=0.28$ y -0.47 para LEG y OLE respectivamente). La nula asociación entre el volumen de producción de LEG y el crecimiento poblacional se asocia a que en los últimos años, la importancia de las LEG como fuente proteica ha disminuido, un ejemplo de ello, es lo que actualmente sucede con el frijol. En esta especie, en un lapso de 11 años (entre 1994 y 2005) su consumo per cápita se redujo en 3.2 kilos pasando de 15 a $11.8 \mathrm{~kg} /$ persona/año (Muñoz-Velázquez et al., 2009). La reducción en el consumo de frijol se asocia con los cambios en la dieta que iniciaron a partir de 1980 cuando la mejora económica favoreció el acceso a la proteína de origen animal (carne, leche y huevos) (Moreno-Altamirano et al., 2014; Soria y Palacios, 2014). La correlación estimada entre la producción de OLE y el aumento poblacional es atípica, 
ya que aun cuando actualmente se ingiere más grasa animal que vegetal, esta preferencia por sí sola no explica la relación negativa estimada entre la producción de OLE y el crecimiento de la población (-0.47). Por lo tanto, la disminución en la producción de OLE de 1980 a la fecha, también podría estar asociada con el estado que guarda el cultivo de soya en México. En 1994 la producción de soya en el Noroeste de México y particularmente en los estados de Sonora y Sinaloa fue severamente afectada por el ataque de mosquita blanca, esto causó que en los próximos años la producción de esta OLE desaparecida en varias zonas agrícolas del país (Avilés-González et al., 2004; Ortega-Arenas, 2006). A la fecha, La producción de soya no se ha recuperado; en consecuencia, esta OLE representa 16 por ciento del valor de la importación de alimentos que realiza México (INEGI, 2015b). A pesar de que en el periodo estudiado el volumen de producción de AROyMED incremento 337 por ciento, este parámetro no presentó correlación con el crecimiento poblacional $(\mathrm{r}=0.48)$, esto se debe a que aun cuando las especies de este grupo se consumen en México, una gran parte de su producción se exporta a otros países (Pérez, 2009). No existe duda de que en los últimos 35 años, el incremento de la producción alimentos en México ha sido función del crecimiento poblacional; sin embargo desde el punto de vista agronómico la pregunta que surge es: ¿cuáles son las estrategias que ha permitido incrementar la producción agrícola en México?

\section{Estrategias asociadas con el aumento de la producción agrícola}

En México al igual que en todo el mundo, el aumento de la producción agrícola ha resultado de mejorar el rendimiento por unidad de superficie, aumentar la superficie cultivada y la combinación de ambas estrategias (Tabla 4). Históricamente tanto a nivel mundial como nacional, el incremento de la producción agrícola vía la mejora del rendimiento por unidad de superficie ha sido clave y en México el caso mejor documentado es el del trigo (Fischer y Edmeades, 2010). Aun cuando la mejora del rendimiento ha ayudado a incrementar la producción de varios cultivos, a la fecha todavía hay algunos en donde su producción es función exclusiva de su área sembrada ya que su rendimiento ha permanecido sin cambios. Por ejemplo, con relación a zanahoria, la ASCA (2000) reporta que su rendimiento no ha cambiado desde 1980 (22.3 ton/ha), por lo tanto, su abasto nacional ha sido cubierto incrementando 500 por ciento su superficie aun cuando su potencial de rendimiento es 64.3 ton/ha (Sosa et al., 2013). Un caso similar ocurre con el frijol en el que su rendimiento en los últimos 14 años ha permanecido sin cambios (FIRA, 2015); aun cuando es posible 
obtener rendimientos de 1.6 ton/ha, el promedio nacional es 0.8 ton/ha (Lara-Flores, 2015). Considerando esta situación, la pregunta a responder es ¿cuáles son grupos de cultivos en los que el rendimiento poco ha cambiado?

\section{Mejora del rendimiento por unidad de superficie}

La asociación estimada entre la producción y el rendimiento de CER $(\mathrm{r}=0.94)$, indica que entre 1980 y 2015, la cantidad de CER producida ha sido función exclusiva de aumentar el rendimiento por unidad de superficie; esto es congruente con lo reportado para trigo en Inglaterra, maíz en EUA y arroz en Japón (Favell, 2010; Fischer y Edmeades, 2010; Jaggard et al., 2010; Phillips, 2010). Para ejemplificar como la mejora del rendimiento ha ayudado a aumentar la producción de CER, en la Figura 1 se presenta los cambios ocurridos en el área cultivada, rendimiento y volumen de producción de trigo durante 55 años en México. Como se puede apreciar, entre 1960 y 2015 la superficie sembrada de trigo se redujo 5.3 por ciento y el rendimiento incremento 320 por ciento pasando de 1.62 a 5.2 ton/ha, esto indica que el aumento de 317 por ciento logrado en la producción de este cereal ha sido función exclusiva de mejorar su rendimiento. La mejora del rendimiento de CER en México y particularmente con relación a trigo y maíz se asocia con la investigación que desde 1950 realiza el CIMMYT en donde fue pionero Norman E. Borlaug y por el cual ganó el premio Nobel en 1970. Esto ha hecho posible que hoy en varias zonas del país se obtengan rendimientos de trigo, maíz y arroz $(8.0,10.2$ y 10.0 ton/ha, respectivamente) (Salcedo-Aceves, 2006; Figueroa-Lopez et al., 2010; MontoyaLeobardo y Armenta-Bojórquez, 2010) superiores la media mundial (2.90, 4.2 .9 y 5.0 , ton/ha, respectivamente) (FAO, 2013).

\section{Aumento del área sembrada}

A diferencia de lo que ha ocurrido en CER, el aumento de la producción en LEG ha estado asociada exclusivamente con incrementar su área cultivada $(\mathrm{r}=0.76)$. Este hecho es preocupante ya que aquí se ubica el frijol el cual desde tiempos ancestrales es pilar de la alimentación Mexicana (CelisVázquez et al., 2010) y hoy con 1.77 millones ha representa 92 por ciento del área cultivada de este grupo; 7.6 por ciento de la superficie restante se destina para producir arvejón, haba, garbanzo y lenteja (SIAP, 2016). Este hecho sugiere que de todas las especies LEG que actualmente se cultivan en México, es en el frijol donde en los próximos años se deberá destinar recursos para hacer investigación y establecer infraestructura que ayude a mejorar su potencial productivo. 
Figura 1: Área cultivada (a), rendimiento (b) y volumen de producción (c) de trigo en México
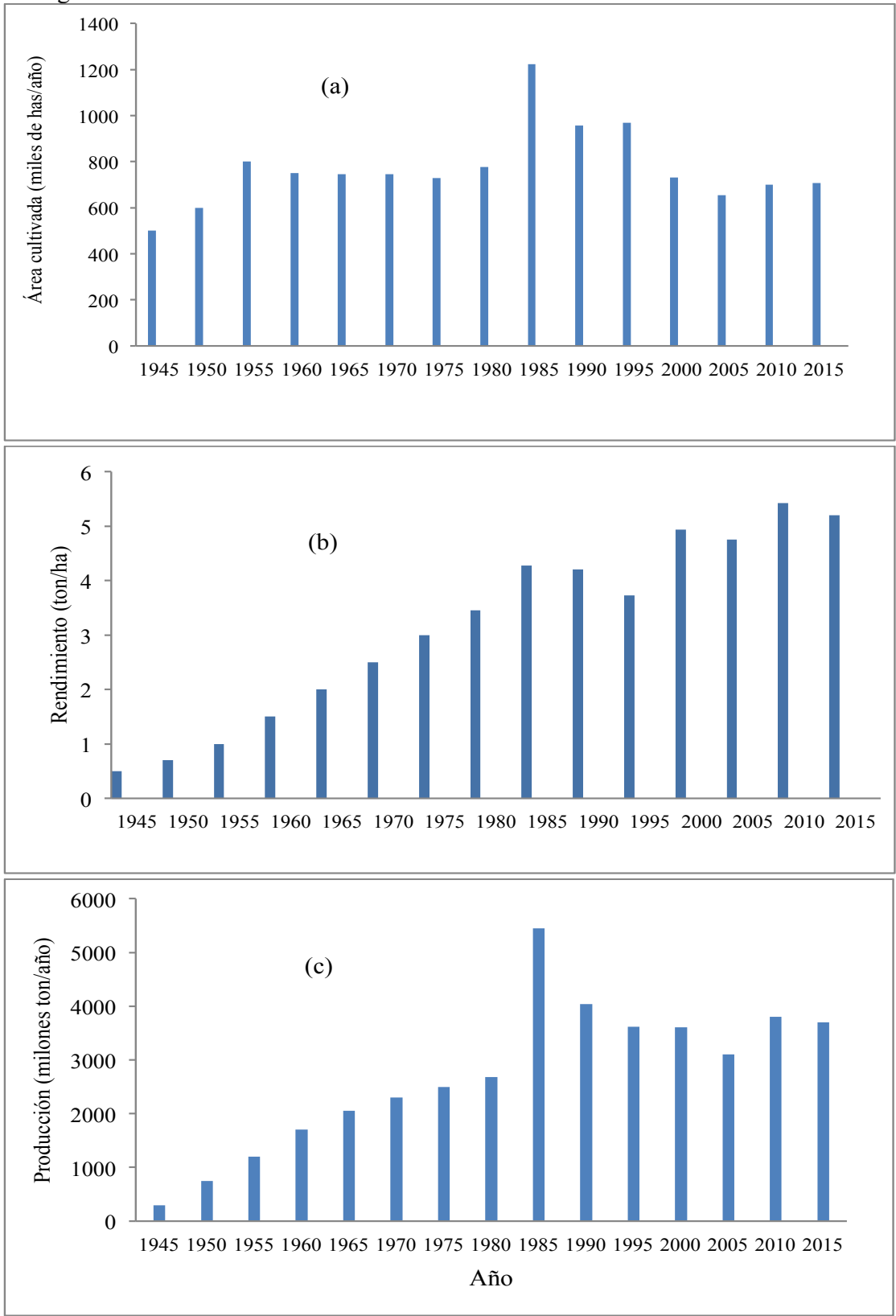

Fuente: los datos de 1945 a 1980 fueron tomados de Bourlag et al. (1982); y de 1980 a 2015 , se utilizó la información del SIAP (2017). 


\section{Incremento del rendimiento y área sembrada}

Como se puede apreciar en la Tabla 4, el aumento en el volumen de producción de OLE, HOR, FRU, IND y AROYMED ha resultado de incrementar su rendimiento y área cultivada. En HOR y FOR el incremento de la producción entre 1980 y 2000 se debió al que su superficie cultivada casi se duplico al pasar de 0.387 a 0.623 y de 2.42 a 4.807 millones de hectáreas para HOR y FOR, respectivamente (Tabla 2). Sin embargo, después de 2000, el área de cultivo de ambos grupos poco incrementó y el aumento de la producción se asoció sólo con la mejora del rendimiento el cual pasó de 17.2 a 23.4 ton/ha en HOR y de 14.82 a 17.7 ton/ha en FOR. La información generada para OLE debe tomarse con cautela ya que aunque los cálculos sugieren que el aumento de su producción se asocia con el incremento del rendimiento y área sembrada $(r=0.80$ y 0.91 , respectivamente), la alta fluctuación de los valores de estos parámetros a través del periodo estudiado indican una baja confiabilidad (Tabla 2).

\section{Escenario de la producción agrícola para los próximos 35 años}

Aun cuando está bien definido como crecerá la población Mexicana, a la fecha no existe una proyección de la demanda total de alimentos que el incremento demográfico impondrá y solo existe una estimación parcial para los productos de origen animal (Salazar-Adams et al., 2006). Considerando esta situación, en la Tabla 5 se presenta la demanda de alimentos vegetales proyectada para los siguientes 35 años.

De acuerdo con el cálculo realizado, para 2050, en México se necesitarán producir casi 289.87 millones ton/año de productos agrícolas. Considerando que no existe más tierra de cultivo, el rendimiento de los cultivos debe aumentar de 12.6 ton/ha en 2010 hasta 15.4 ton/ha en 2050 (Tabla 6).

Ante este panorama, la pregunta que surge: $¿$ es factible incrementar la producción exclusivamente mediante la mejora del rendimiento? Para responder esta pregunta, en la Figura 2 se presenta la ganancia en rendimiento lograda en seis grupos de cultivos en los últimos 35 años. Los resultados sugieren que en CER, HOR, FRU, FOR, IND y LEG será posible cubrir su demanda incrementando su rendimiento, esta afirmación se basa en que la ganancia en rendimiento lograda entre 1980 y 2014 para los grupos antes mencionados (44, 319, 97, 174, 242 y $32 \mathrm{~kg} / \mathrm{ha} /$ año, respectivamente) es mayor a la que se estima es suficiente para asegurar su abasto $(24,145,87$, 170,208 y $5 \mathrm{~kg} / \mathrm{ha} /$ año, respectivamente) (Tabla 6). 
Tabla 5: Proyección del volumen de demanda esperada de ocho grupos de cultivos en México entre 2015-2050

\begin{tabular}{lrrrrrrr}
\hline & $\begin{array}{r}\text { Disponibilidad } \\
\text { alimentos 2010 }\end{array}$ & 2014 & 2015 & 2020 & 2030 & 2040 & 2050 \\
\cline { 5 - 9 } & (kg/habitante año) & \multicolumn{7}{c}{ Millones ton/año } \\
\hline Cereales & 317.0 & 36.59 & 36.14 & 37.47 & 40.42 & 42.79 & 44.38 \\
Leguminosas & 12.1 & 1.49 & 1.38 & 1.43 & 1.54 & 1.63 & 1.69 \\
Oleaginosas & 10.0 & 2.61 & 1.14 & 1.18 & 1.27 & 1.35 & 1.40 \\
Hortalizas & 118.1 & 15.57 & 13.46 & 13.96 & 15.06 & 15.94 & 16.53 \\
Frutales & 162.2 & 18.74 & 18.49 & 19.17 & 20.68 & 21.90 & 22.71 \\
Forrajes & 953.0 & 62.08 & 108.64 & 112.64 & 121.51 & 128.66 & 133.42 \\
Industriales & 497.9 & 62.68 & 56.76 & 58.85 & 63.48 & 67.22 & 69.71 \\
AROyMED & 0.2 & 0.05 & 0.02 & 0.02 & 0.03 & 0.03 & 0.03 \\
Total & 2178 & 199.82 & 236.04 & 244.73 & 263.99 & 279.52 & 289.87 \\
\hline Fonnyyyyyy
\end{tabular}

Fuente: Valores estimados a partir de información de la CONAPO (2006) y el SIAP (2016).

Tabla 6: Incremento del rendimiento necesario para satisfacer el volumen de demanda esperada de ocho grupos de cultivos en México entre 2015-2050

Superficie

Grupo (millones ha) Rendimiento $(\mathrm{kg} / \mathrm{ha})$

\begin{tabular}{lrrrrrrr}
\hline & & 2015 & 2020 & 2030 & 2040 & 2050 & Anual \\
\hline Cereales & 9.705 & 3.7 & 3.9 & 4.2 & 4.4 & 4.6 & 24.3 \\
Leguminosas & 1.746 & 0.8 & 0.8 & 0.9 & 0.9 & 1.0 & 5.1 \\
Oleaginosas & 0.702 & 1.6 & 1.7 & 1.8 & 1.9 & 2.0 & 10.6 \\
Hortalizas & 0.604 & 22.3 & 23.1 & 24.9 & 26.4 & 27.4 & 145.3 \\
Frutales & 1.385 & 13.4 & 13.8 & 14.9 & 15.8 & 16.4 & 87.0 \\
Forrajes & 4.156 & 26.1 & 27.1 & 29.2 & 31.0 & 32.1 & 170.3 \\
Industriales & 1.778 & 31.9 & 33.1 & 35.7 & 37.8 & 39.2 & 208.0 \\
AROyMED & 0.031 & 0.7 & 0.8 & 0.8 & 0.9 & 0.9 & 4.8 \\
Total & 20.106 & 12.6 & 13.0 & 14.1 & 14.9 & 15.4 & 81.9 \\
\hline Nof Arial
\end{tabular}

Nota: Área agrícola cosechada durante el año 2010.

Fuente: Valores estimados con base en datos del SIAP (2016) y CONAPO (2006).

En CER, el rendimiento estimado es 4.6 ton/ha, este valor supera al que la FAO (2009) estima debe aumentar el rendimiento de CER (4.3 ton/ha) en el mundo. Con respecto a LEG, la ganancia en rendimiento lograda en el periodo estudiado fue $4.8 \mathrm{~kg} / \mathrm{ha} / \mathrm{año}$, este valor es menor al requerido para asegurar su abasto hacia $2050(5.1 \mathrm{~kg} / \mathrm{ha} / \mathrm{año})$. La baja productividad de LEG en México se asocia a que aunque hay zonas productoras de frijol en Sinaloa y Durango en donde se obtienen rendimientos (1.7 y 1.9 ton/ha) (López-Salinas, 2011) que superan la media nacional (0.72 ton/ha) (SIAP, 2016), a la fecha todavía hay regiones donde la productividad es menor de 0.5 ton/ha (SIAP, 2016). 
Figura 2: Tendencia de la mejora del rendimiento (ton/ha/año) en seis grupos de cultivo durante 35 años en México
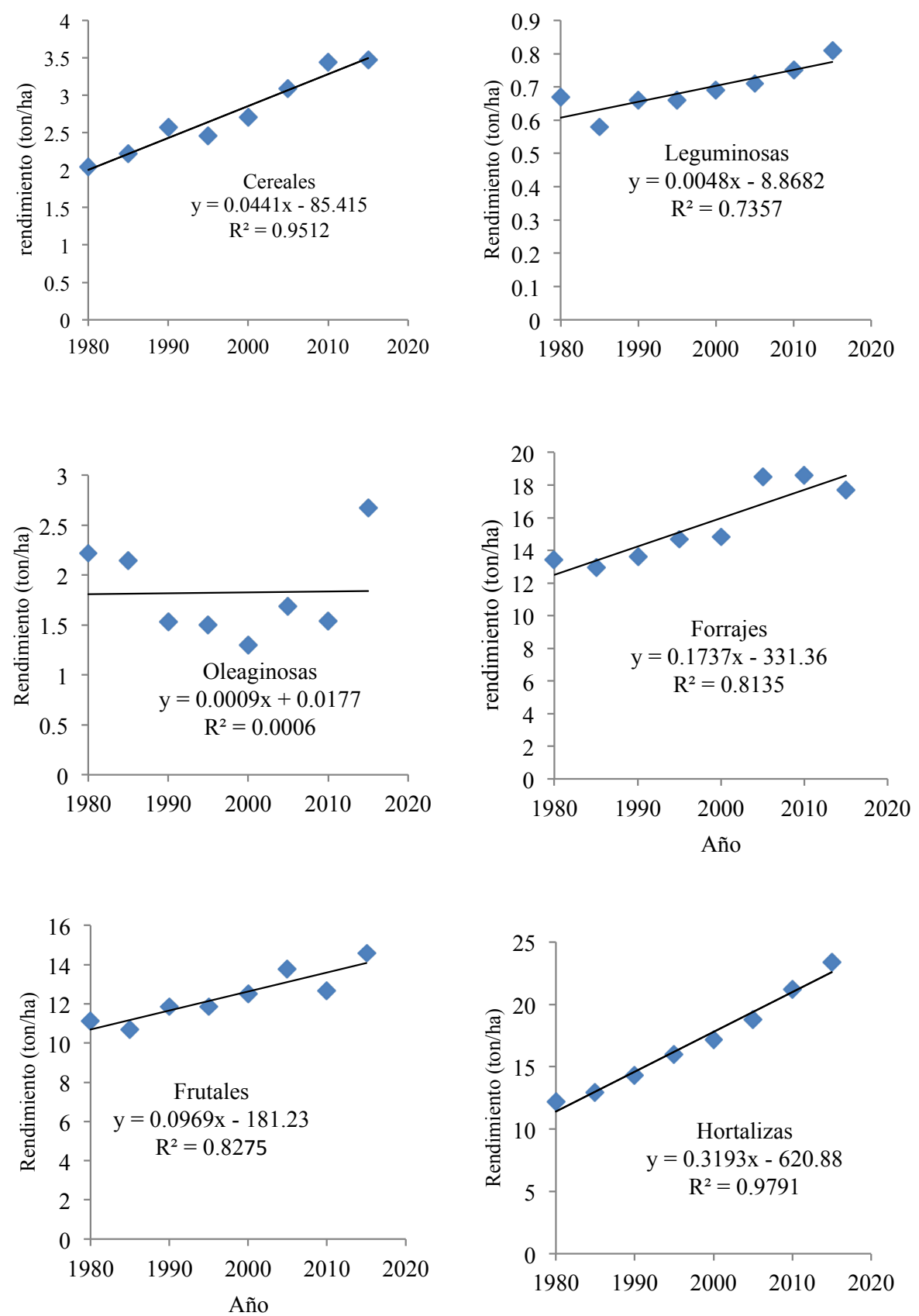

Fuente: Figura realizada con base en datos del SIAP (2016). 
El frijol es un cultivo de temporal, de ahí que a futuro para asegurar su abasto será necesario destinar recursos a investigación que permita generar tecnología para la producción de frijol bajo este sistema. Actualmente el INIFAP trabaja en la obtención de cultivares resistentes a sequía y a enfermedades (Anaya-López et al., 2015) y se espera que en el corto plazo se cuente con variedades que permitan mejorar su rendimiento. En OLE, la mejora del rendimiento $(0.9 \mathrm{~kg} / \mathrm{ha} / \mathrm{año})$ fue inferior al requerido para asegurar su abasto $(10.6 \mathrm{~kg} / \mathrm{ha} / \mathrm{año})$, esta situación ya está prevista y hoy día se realiza investigación para generar practicas agronómicas que ayuden a mejorar el rendimiento de algunas de las especies de este grupo (algodón, cártamo y girasol); además se evalúa la posibilidad de introducir nuevas OLE, tal es el caso de la canola (Inzunza-Ibarra et al., 2014). De acuerdo con la información presentada, de 1980 a la fecha, la mejora del rendimiento de los cultivos es evidente; particularmente después del año 2000 (Tabla 2) cuando se comenzó a utilizar el riego por goteo y la agricultura protegida. Sin embargo, debido a que todavía existen cultivos en donde la ganancia de rendimiento es baja, hay incertidumbre con relación a si la agricultura de México está preparada para cubrir la demanda futura de alimentos. El incremento poblacional para los 35 años siguientes se ha calculado con base en diferentes escenarios (CONAPO, 2006) y zonas del país (Azuz-Adeath y Rivera-Arriaga, 2007). Sin embargo, con relación a la demanda y producción de alimentos, a la fecha no existen estimaciones; de acuerdo con Camberos-Castro (2000), esto se debe a que es difícil hacer estas proyecciones y siempre tendrán cierto grado de especulación porque se deben hacer algunas suposiciones como las realizadas en este trabajo. Los resultados de este este análisis indican que la agricultura mexicana cuenta con la capacidad y los recursos para cubrir la demanda de alimentos. Esta afirmación contradice a quienes sostienen que México carece de autosuficiencia alimentaria y por ende tiene que recurrir a la importación de alimentos (Flores-Pérez et al., 2012; López, 2015). A este respecto, la importación de alimentos no significa que nuestro país tiene dependencia alimentaria ya que todos los países del mundo en alguna medida recurren a esta para abastecerse de los alimentos que no pueden producir en alguna época del año o bien porque su volumen producido es menor a la demanda de su población. Para medir la autosuficiencia alimentaria de un país, la FAO (2009) recomienda realizar un balance energético entre los alimentos que se producen y consumen internamente, los que se exportan a otros países y los que se importan del extranjero (FAO, 2009). Este método es laborioso, de ahí que una alternativa práctica consiste en analizar la balan- 
za comercial de exportación e importación de alimentos; suponiendo que si el balance es positivo se asume que existe autosuficiencia alimentaria; por el contrario, un saldo negativo significa que existe déficit en la producción de alimentos. En la Tabla 7 se presenta el balance de la importación y exportación de alimentos que el INEGI (2015b) reporta se realizó en México durante 2014 y 2015. Como se puede apreciar en 2014 y 2015 la balanza comercial de alimentos de México presentó saldo positivo (69.9 y 324.9 millones de dólares para 2014 y 2015, respectivamente).

Este resultado es una prueba más que se sustenta la afirmación de que México se produce más alimentos de los que demanda la población y es autosuficiente desde el punto de vista alimentario. Es una realidad que la producción nacional de CER y LEG no es suficiente para cubrir la demanda de la población y hoy el maíz junto con soya y trigo representan 44 por ciento de la importación total de alimentos. Este hecho ha conducido a varios a afirmar que en México existe déficit alimentario lo cual es erróneo ya que de acuerdo con la balanza comercial 2015, tan solo el ingreso de la exportación de HORT (576.3 millones de dólares) fue 87 por ciento mayor que lo que costó importar CER (285.9 millones de dólares). De acuerdo con Borlaug et al. (1982) la exportación de HORT es una estrategia económica que desde 1970 el gobierno mexicano impulsó para generar divisas y empleos; por lo tanto, aun cuando la balanza comercial de alimentos 2015 reporta una ganancia de 324.2 millones de dólares, el costo de ello es la importación de granos básicos para cubrir la demanda de la población. El balance positivo de la balanza comercial del año 2014 y 2015 confirma lo que reporta la FAO (2014) con relación a la disponibilidad de alimentos en México, este organismo internacional afirma que este país produce suficiente alimento, sin embargo su precio elevado como resultado del costo de su traslado a las localidades limita su adquisición aun cuando este se encuentre disponible y aseguran que este problema no es por falta de producción sino de acceso. Para mejorar el acceso a los alimentos en México, la FAO (2013) sostiene que además de incrementar su producción; también se debe trabajar en (1) mejorar la red de distribución y transporte de alimentos: (2) reducir la tasa de desempleo; (3); mejorar el salario; y (4) regular el costo de la canasta básica. Esto significa que en materia de seguridad alimentaria, producir alimentos es solo una parte de la ecuación y la otra que quizás es la más importante es distribuirlos con equidad; esto último es la más grande faya no solo de México sino de muchos otros países el mundo. 
La disponibilidad de alimentos en México: un análisis de la producción agrícola ... /A. SOSA-BALDIVIA y G. RUIZ

Tabla 7: Balance de la importación y exportación de alimentos realizada en México durante 2014 y 2015 (millones de dólares)

\begin{tabular}{|c|c|c|c|c|c|c|}
\hline \multirow[t]{2}{*}{ Concepto } & \multicolumn{3}{|c|}{ Año 2014} & \multicolumn{3}{|c|}{ Año 2015} \\
\hline & EXP & IMP & Balance & EXP & IMP & Balance \\
\hline Productos de origen animal & 120.8 & 177.7 & -56.9 & 184.1 & 167 & 17.1 \\
\hline Ganado vacuno & 46.2 & 4.5 & 41.7 & 69.3 & 3.1 & 66.2 \\
\hline Leche y sus derivados & 0.0 & 71.7 & -71.7 & 0.0 & 62.2 & -62.2 \\
\hline Huevo & 0.0 & 11.2 & -11.2 & 0.0 & 17.4 & -17.4 \\
\hline Vísceras de animales & 0.0 & 17.3 & -17.3 & 0.0 & 20.4 & -20.4 \\
\hline Pescados y Mariscos & 48.3 & 73.0 & -24.7 & 69.5 & 63.9 & 5.6 \\
\hline Camarón congelado & 26.3 & 0.0 & 26.3 & 45.3 & 0.0 & 45.3 \\
\hline Cereales & 33.6 & 329.6 & -296 & 15.2 & 301.1 & -285.9 \\
\hline Maíz & 3.4 & 186.4 & -183 & 4.5 & 165.3 & -160.8 \\
\hline Trigo & 30.2 & 90.2 & -60 & 10.7 & 89.1 & -78.4 \\
\hline Arroz & 0.0 & 38.4 & -38.4 & 0.0 & 30.0 & -30.0 \\
\hline Sorgo & 0.0 & 9.6 & -9.6 & 0.0 & 5.0 & -5.0 \\
\hline Otros & 0.0 & 5.0 & -5.0 & 0.0 & 11.7 & -11.7 \\
\hline Leguminosas & 19.7 & 5.0 & 14.7 & 11.2 & 7.0 & 4.2 \\
\hline Frijol & 5.5 & 5.0 & 0.5 & 2.7 & 7.0 & -4.3 \\
\hline Garbanzo & 14.2 & 0.0 & 14.2 & 8.5 & 0.0 & 8.5 \\
\hline Oleaginosas & 0.0 & 257.3 & -257.3 & 0.0 & 210.7 & -210.7 \\
\hline Soya & 0.0 & 162.1 & -162.1 & 0.0 & 146.9 & -146.9 \\
\hline Colza & 0.0 & 72.3 & -72.3 & 0.0 & 32.7 & -32.7 \\
\hline Otras & 0.0 & 22.9 & -22.9 & 0.0 & 31.1 & -31.1 \\
\hline Industriales & 26.9 & 57.2 & -30.3 & 36.6 & 41.3 & -4.7 \\
\hline Café & 22.1 & 0.0 & 22.1 & 30.4 & 0.0 & 30.4 \\
\hline Tabaco & 0.8 & 13.1 & -12.3 & 0.9 & 11.4 & -10.5 \\
\hline Algodón & 4.0 & 44.1 & -40.1 & 5.3 & 29.9 & -24.6 \\
\hline Frutas & 283.8 & 70.2 & 213.6 & 352 & 75.7 & 276.3 \\
\hline Plátano & 10.7 & 0.0 & 10.7 & 11.4 & 0.0 & 11.4 \\
\hline Mango & 2.8 & 0.0 & 2.8 & 2.7 & 0.0 & 2.7 \\
\hline Aguacate & 134.6 & 0.0 & 134.6 & 156.4 & 0.0 & 156.4 \\
\hline Cítricos & 24.7 & 0.0 & 24.7 & 29.9 & 0.0 & 29.9 \\
\hline Melón, papaya y sandia & 15.2 & 0.0 & 15.2 & 18.3 & 0.0 & 18.3 \\
\hline Fresas frescas & 37.6 & 0.0 & 37.6 & 49.2 & 0.0 & 49.2 \\
\hline Frutas frescas y secas & 56.6 & 23.3 & 33.3 & 83.9 & 33.7 & 50.2 \\
\hline Uvas y pasas & 1.6 & 13.3 & -11.7 & 0.2 & 9.7 & -9.5 \\
\hline Manzanas, peras y membrillos & 0.0 & 33.6 & -33.6 & 0.0 & 32.3 & -32.3 \\
\hline Hortalizas & 539.4 & 2.9 & 536.5 & 584.7 & 7.1 & 577.6 \\
\hline Jitomate & 167.7 & 0.0 & 167.7 & 197.2 & 0.0 & 197.2 \\
\hline Chiles y pimientos frescos y secos & 137.0 & 2.9 & 134.1 & 141.8 & 7.1 & 134.7 \\
\hline Cebollas y ajos & 25.4 & 0.0 & 25.4 & 22.7 & 0.0 & 22.7 \\
\hline Pepino & 62.3 & 0.0 & 62.3 & 54.6 & 0.0 & 54.6 \\
\hline Otras & 147.0 & 0.0 & 147.0 & 168.4 & 0.0 & 168.4 \\
\hline Varios & 47.9 & 102.2 & -54.3 & 48.6 & 98.3 & -49.7 \\
\hline Otros productos agropecuarios & 37.8 & 76.1 & -38.3 & 40.1 & 77.3 & -37.2 \\
\hline Semillas para siembra & & 26.1 & -26.1 & 0.0 & 21.0 & -21.0 \\
\hline Flores & 1.9 & 0.0 & 1.9 & 1.6 & 0.0 & 1.6 \\
\hline Miel & 8.2 & 0.0 & 8.2 & 6.9 & 0.0 & 6.9 \\
\hline Total & 1072.1 & 1002.1 & 70.0 & 1232.4 & 908.2 & 324.2 \\
\hline
\end{tabular}

EXP $=$ Exportaciones; IMP $=$ Importaciones

Fuente: los cálculos se realizaron con base en la información reportada por el INEGI, (2015b). 


\section{Conclusiones}

Para el año 2050 en México solo se dispondrá de 0.18 has de tierra cultivable para producir los alimento que demanda una persona al año. Debido a que no existe más tierra cultivable para asegurar el abasto de alimentos nacional rumbo al 2050 el rendimiento medio los ocho grupos cultivos debe de incrementarse en 2.8 toneladas pasando de las 12.6 ton/ha actuales a 15.4 ton/ha. Las mejoras en el rendimiento realizadas en los últimos 30 años en CER, HOR, FRU, FOR e IND indican que aun manteniendo la superficie cultivada del 2010 será posible asegurar el suministro de los cultivos que comprenden este grupo a la población. Es en LEG y OLE en donde se debe de realizar investigación para generar tecnología e invertir en infraestructura agrícola que permita incrementar los rendimientos como medida que asegure su suministro en México.

\section{EPILOGO}

\section{La antología de las fresas, una explicación simple al problema de alimentación en México}

La idea de escribir este artículo fue producto de una discusión que sostuve con un amigo holandés que visitó México en 2012. El debate inició cuando él menciono que nuestro país carece de autosuficiencia alimentaria; yo nunca he estado de acuerdo con ello, así que decidí dar mi opinión. Como no tenía bases para sustentar mi postura, ingeniosamente utilicé lo que más tarde nombré como "la antología de las fresas" y que improvise en ese momento. Históricamente, Irapuato Guanajuato, se considera la capital de las fresas en México; irónicamente, aun cuando mi lugar de origen (Ticumán Morelos) está más cerca de esta zona agrícola que el de mi amigo (Wageningen Holanda), no fue hasta la edad de 23 años cuando por primera vez comí fresas frescas; en tanto que él las comió desde niño. Considerando este hecho, la pregunta que hice a mi amigo fue: si en México hay mayor disponibilidad de fresas que en Holanda ¿cómo explicas que tú las comes desde niño, mientras que yo solo las puedo comer hasta hoy que trabajo como profesionista? La respuesta es simple y se llama acceso. Este concepto desde el punto de vista alimentario se asocia con la disponibilidad monetaria que tiene un individuo, familia, estado y/o país para adquirir alimentos independientemente del precio. Los plátanos no se producen en Suiza y sin embargo los consumen; los emiratos árabes no producen hortalizas y sin embargo las consumen; derivado de esto, la pregunta que surge es: ¿cómo estos países logran esto? Este hecho es posible porque la 
población de estos países tienen el poder adquisitivo para acceder a estos alimentos al precio que sea; por lo tanto, es evidente que mi amigo pudo comer fresas porque aun cuando en Holanda su disponibilidad es menor que en México, su padre al ser profesionista ganaba lo suficiente para comprar fresas aunque estas fuesen caras; en tanto mi padre con su salario de jornalero nunca pudo comprarlas aun cuando estaban disponibles en el mercado local y eran relativamente baratas. La charla finalizó y aunque mi amigo me dio la razón; muy en el fondo sabía que mi explicación carecía de bases sólidas; por lo que considerando que la situación alimentaria de México es un tema de debate me propuse buscar la respuesta verdadera basada en datos duros. Como versión preliminar de este trabajo estuvo lista en 2013, la envié a un concurso organizado por la UABC en Mexicali Baja California en donde el premio para los tres primeros lugares era publicar su trabajo. No gane el concurso y al no contar con presupuesto para publicarlo decidí esperar por dos razones; la primera se debió a que como los cálculos los realice por lustro necesitaba el resultado del censo poblacional, agrícolas y ganaderos 2015 para completarlo; y la segunda fue encontrar financiamiento para poder pagar el arbitraje y publicación del trabajo. La versión final la envié a la revista Archivos Latinoamericanos de Nutrición en Junio de 2016 y aunque hubo interés, al final el editor me recomendó publicarlo en una revista científica mexicana para su mejor difusión. De acuerdo con la información presentada en este trabajo, está claro que el problema alimentario en México no es disponibilidad sino de acceso a los alimentos; esto significa que aun cuando el alimento esté disponible, si una persona no cuenta con solvencia económica no tendrá la capacidad para adquirirlos tal como en el pasado me sucedió con las fresas. Es cierto que actualmente nuestro país destina 301.1 y 210.7 millones de dólares para importar CER y OLE, respectivamente; sin embargo, aun con ello nuestra agricultura logra un excedente monetario de 324.2 millones de dólares debido a que tan solo el ingreso derivado de la exportación de frutas (352 millones de dólares) y verduras (584.7 millones de dólares) rebasa ampliamente a la cantidad monetaria que se destina a la importación de granos básicos (511 millones de dólares gastados en importación de granos y oleaginosas vs. 936.7 dólares de ganancia en la exportación de frutas y verduras). Estos cálculos se hicieron con base en la balanza comercial que reporta el INEGI para 2015; así que la única forma de que estos sean erróneos es que la fuente este equivocada. 


\section{REFERENCIAS BIBLIOGRÁFICAS}

Agrios, N.G., 1998, Plant Pathology, Academic Press Inc. Florida, USA, 703 p.

Anaya-Lopez, L.J., Silva-Rosales, L., Moreno-Tavera, V., Espejel, F. y AcostaGallegos, J.A., 2015, "Retos y oportunidades en la selección asistida de frijol resistente a BCMV y BCMNV en México. I. Dimensión del problema", en Revista Mexicana de Ciencias Agrícolas, núm. 6, pp. 453-465.

ASCA, 2000, "La zanahoria mexicana, una hortaliza con crecimiento sostenido", en Claridades Agropecuarias, núm. 79, Apoyos y Servicios a la Comercialización Agropecuaria ,pp. 1-16.

Avilés-González, C.M., Nava-Camberos, U., Garzón-Tiznado, J.A., Wong-Pérez, J.J. y Pérez-Valdez, J.J., 2004, Manejo integrado de la mosquita blanca Bemisia sp., en tomate para consumo fresco, INIFAP/CIRNO, Campo experimental del valle de Culiacán, Folleto Técnico núm. 28, Culiacán, Sinaloa, México.

Azuz-Adeath, I. y Rivera-Arriaga, E., 2007, "Estimación del crecimiento poblacional para los estados costeros de México", en Papeles de Población, núm. 51, pp. 187-211.

Borlaug, E.N., Hanson, H. y Anderson, G.R., 1982, Wheat in the third world, Westview Press Inc. Colorado, USA.

Camberos-Castro, M., 2000, "La seguridad alimentaria en México en el año 2030", en Ciencia Ergo Sum, núm. 7, pp. 49-55.

Castellanos-Ramos, J.Z. y Borbón-Morales, C., 2009, "Panorama de la horticultura protegida en México", en Castellanos R.J.Z. (ed.), Manual de producción de tomate en invernadero, INTAGRI, Celaya, Guanajuato, México, pp. 1-18.

Celis-Velázquez, R., Peña-Valdivia, C.B., Luna-Cavazos, M. y Aguirre, J.R., 2010, "Seed morphological characterization and reserves used during seedling emergency of wild and domesticated common bean (Phaseolus vulgaris L.)", en Rev. Fac. Agron, núm. 27, pp. 61-87.

CONAPO, 2006, Proyecciones de la población de México 2005-2050, Consejo Nacional de Población. México, 30 p.

FAO, 2009, "La agricultura mundial en la perspectiva del año 2050", en Cómo alimentar al mundo en 2050, Secretaría del Foro de Alto Nivel de Expertos, Italia, Roma, pp. 4.

FAO, 2013, Panorama de la seguridad alimentaria y nutricional en México, México. $240 \mathrm{p}$.

FAO, 2014, "La alimentación en América Latina y el Caribe", en Anuario estadístico de la FAO, Oficina Regional para América Latina y el Caribe. Santiago, Chile. 179 p.

Favell, R., 2010, "Knowledge and technologies for sustainable intensification of food production", en New Biotechnology, núm, 27, pp. 505-516. 
Figueroa-López, P., Félix-Fuentes, J.L., Fuentes-Dávila, G., Valenzuela-Herrera, V., Chávez-Villalba, G. y Mendoza-Lugo, J.A., 2010, "CIRNO C2008 nueva variedad de trigo cristalino con alto rendimiento potencial para el estado de Sonora", en Revista Mexicana de Ciencias Agrícolas, núm. 51, pp. 745-749.

Financiera Rural, 2008, La producción de hortalizas en México, Dirección General Adjunta de Fomento y Promoción de Negocios, México.

FIRA, 2015, “Panorama alimentario: frijol 2015”, Dirección de Investigación y Evaluación Economica y Sectorial, México, pp. 31.

Fischer, R.A. y Edmeades, G., 2010, "Breeding and cereal yield progress", en Crop Science, núm. 50, pp. 85-98.

Flores-Pérez, B., Vázquez-Ortiz, P. y Quintero-Soto, L.M., 2012, “Soberanía, seguridad, autosuficiencia o crisis alimentaria? Caso de México y la región de África. Problema básico en salud y calidad de vida", en Revista Digital Universitaria, núm. 13, pp. 1-19.

INEGI, 2015a, "Principales resultados de la encuesta intercensal 2015", Instituto Nacional de Estadística y Geografía (INEGI), Aguascalientes, México. pp. 122.

INEGI, 2015b, Balanza comercial de mercancías de México. Información revisada enero, INEGI. Aguascalientes, México. 61 p.

INEGI-SAGARPA, 2015, "Nota técnica Encuesta nacional agropecuaria 2014", en Conociendo el campo de México, Instituto Nacional de Estadística Geografía y Secretaría de Agricultura, Ganadería, Desarrollo Rural, Pesca y Alimentación, Aguascalientes, México, pp. 20.

Inzunza-Ibarra, A.M., Catalán-Valencia, E.A., Villa-Castorena, M.M., SánchezCohen, I., Sifuentes-Ibarra, E. y Román-López, A., 2014, “Modelos de predicción de rendimiento de canola en función del contenido de humedad en el suelo", en Revista Fitotecnia Mexicana, núm. 37, pp. 49-58.

Jaggard, W.K., Qui, A. y Ober, E.S., 2010, "Possible changes to arable crop yields by $2050 ”$, en Phil. Trans. R. Soc. núm. 365, pp. 2835-2851.

Lara-Flores, M., 2015, “El cultivo de frijol en México", en Revista Digital Universitaria UNAM, vol. 16, núm. 2, pp. 1-11.

López, S.R., 2015, "Pobreza y seguridad alimentaria: el caso de México", en Revista CIS, núm. 18, pp. 24-54.

López-Salinas et al., 2011, "Estabilidad de rendimiento en genotipos mesoamericanos de frijol de grano negro en México", en Revista Mexicana de Ciencias Agrícolas, núm. 2, pp. 29-40.

Macías, M. A., 2010, “Competividad de México en el mercado de frutas y hortalizas de Estados Unidos de América”, en Agroalimentaria, núm. 16: 31-48.

Martínez-Jasso, I. y Villezca-Becerra, P.A., 2005, “La alimentación en México: Un estudio a partir de la encuesta nacional de ingresos y gastos y de las hojas de balance alimenticio de la FAO”, en Ciencia UANL, núm. 8, pp. 196-208.

Montoya-Leobardo, G. y Armenta-Bojorquez, D.A., 2010, "Reflexiones sobre el impacto del cultivo de maíz en Sinaloa”, en Ra Ximhai, núm. 6, pp. 69-72. 
Moreno-Altamirano, L. et al., 2014, "La transición alimentaria y la doble carga de malnutrición: cambio en los patrones alimentarios de 1961 a 2009 en el contexto socioeconómico mexicano", en Archivos Latinoamericanos de Nutrición, núm. 64, pp. 231-240.

Muñoz-Velázquez, E.E., Rubio-Hernández, D., Bernal-Lugo, I., Garza-García, R. y Jacinto-Hernández, C., 2009, "Caracterización de genotipos nativos de frijol del estado de Hidalgo, con base a calidad del grano", en Agricultura Técnica en México, núm. 35, pp. 426-435.

Ortega-Arenas, D.L., 2006, "Bioecología de las moscas blancas", en Ortega-Arenas, D.L. (ed.), Moscas blancas temas selectos sobre su manejo, Mundiprensa, México, pp. 1-6.

Ortiz-Hernández, L., Delgado-Sánchez, G. y Hernández-Briones, A., 2006, “Cambios en factores relacionados con la transición alimentaria y nutricional en México", en Gaceta Medica Mexicana, núm. 142, pp. 181-193.

Pérez, C.R., 2009, "Frutas y hortalizas orgánicas de la red de mercados y tianguis orgánicos de México", en Claridades Agropecuarias, núm. 194, pp. 35-45.

Phillips, L.R., 2010, "Mobilizing science to break yield barriers", en Crop Science, núm. 59, pp. 99-108.

Resh, M.H., 1992, Cultivos hidropónicos, Mundi-Prensa. Madrid, España.

Román, S., Ojeda-Granados, C. y Panduro, A., 2013, "Genética y evolución de la alimentación de la población en México", en Revista de Endocrinología y Nutrición, núm. 21, pp. 42-51.

Salazar-Adams, J.A., Cervantes-Escoto, F., Gómez-Cruz, A.M., Mohanty, S. y Malaga, J., 2006, "La demanda de productos pecuarios en México por deciles de ingreso: Proyección al año 2025", en Técnica Pecuaria en México, núm. 1, pp. 41-52

Salcedo-Aceves, J., 2006, Morelos A-98, variedad de arroz para Morelos y otros estados de la república, folleto técnico núm. 21, INIFAP, Centro de Investigación Regional del Centro, Campo Experimental de Zacatepec Morelos, México.

SIAP, 2016, Producción agrícola 2016, Servicio de Información Agrícola y Pecuaria, disponible en http://www.siap.org, consulta 11/02/2016.

Soria, S.G. y Palacios, V.H., 2014, "El escenario actual de la alimentación en México", en Textos y Contextos Porto Alegre, núm. 13, pp.128-142.

Sosa, A., Ruíz, G., Bazante, I., Mendoza, A., Etchevers, J.D., Padilla, J. y Castellanos, J., 2013, "Absorción de nitrógeno, fosforo y potasio en zanahoria cultivada en el Bajío de México", en Informaciones Agronómicas Hispanoamérica, pp. 27-30.

Turrent-Fernández, A. y Moreno-Dahme, R., 1998, "Producción sostenible de alimentos de origen vegetal en el mundo", en Terra Latinoamericana, núm. 16, pp. 93-111.

Urquiza-Fernández, N., 2014, "La seguridad alimentaria en México", en Salud Pública de México, núm. 56, pp. 92-98. 


\section{RESUMEN CURRICULAR DE LOS AUTORES}

\section{Anacleto Sosa Baldivia}

Maestro en Ciencias en Fertilidad de Suelos egresado del Colegio de Postgraduados y que actualmente cursa estudios doctorales en el Centro de Biotecnología Aplicada del Instituto Politécnico Nacional. Desde hace tres años es Investigador en Nutrilite-Amway, en GAO, México; en donde conduce investigaciones para descubrir e incrementar la concentración de fitonutrientes en plantas medicinales y cultivos a nivel global. Su trabajo de investigación es multidisciplinario y ha publicado trabajos relacionados con la Nutrición de Cultivos, Entomología, Fitopatología, Mejoramiento Genético de plantas y Nutrición Humana. Tan solo en 2016 publicó seis artículos in extenso como autor principal los cuales fueron: (1) Will be boron essential on human nutrition (Archivos Latinoamericanos de Nutrición); (2) Será Diabrotica una plaga de importancia económica en chia? (Entomología Mexicana); (3) History of chia as a source of fatty acids Omega 3 around the world (Journal: Crop Research and Fertilizer); (4) Five causes why boron essentiality on humans has not been confirmed: A hypothesis (Journal: Integrative Food, Nutrition and Metabolism); (5) Demanda de macro y micronutrientes en espinaca cultivada en El Llano en Llamas de Jalisco (El Jornalero); y (6) Demanda de N, P y K en zanahoria cultivada en el Bajío de México (El Jornalero). Después de un largo trabajo de investigación de cuatro años, para 2017 registrará la primer variedad de chía (Salvia hispanica L.) de semilla blanca de alto potencial de rendimiento y resistente a enfermedades en todo el mundo.

Dirección electrónica: anacleto.sosa@amway.com

\section{Guadalupe Ruíz Ibarra}

Ingeniero Biotecnólogo egresado del Instituto Tecnológico de Sonora ubicado en Obregón Sonora, México. Actualmente es profesor del Instituto Tecnológico de Tamazula de Gordiano Jalisco, México y para Julio de 2017 iniciará con sus estudios de Maestría en el Centro de Biotecnología Aplicada en el Instituto Politécnico Nacional. Este investigador cuenta con amplia experiencia en la conducción de investigación relacionada con el estudio de micorrizas en sistemas de producción agrícola y fisiología de cultivos. La tesis que en el CIMMYT realizo esta investigadora (Investigación sobre la posibilidad de cambiar las características de fotosíntesis en trigo (Triticum sp.)) fue la base para desarrollar la teoría sobre la importancia de la fotosíntesis que realizan las barbas del trigo en el incremento del 
rendimiento de grano. Es co-autora, editora de estilo y lo más importante compañera de autor principal con quien formó una bonita familia integrada por Carlos, Daniel, Diana, Nadia y Brenda en donde el slogan es que la unión hace la fuerza y por lo tanto la manada jamás se separa.

Dirección electrónica: lupytaruiz@gmail.com

Artículo recibido el 17 de febrero de 2017 y aprobado el 17 de marzo de 2017. 